\title{
Current strategies and challenges in treatment of childhood rhabdomyosarcoma
}

\author{
Natia Esiashvili • Roshan Prabhu • Shannon Kahn • \\ Arnold C. Paulino
}

Received: 8 November 2012 / Accepted: 12 November 2012 /Published online: 29 November 2012

(C) Springer-Verlag Berlin Heidelberg 2012

\begin{abstract}
Rhabdomyosarcoma (RMS) is the most common soft tissue sarcoma in children, affecting very young patients. These tumors often cause significant functional damage because of their aggressive growth pattern. In addition, their metastatic potential can present as a complex and challenging situation. RMS can present in various anatomical sites and often pose significant obstacle in choosing local control modalities. When feasible, surgery plays an important role for initial diagnosis and complete tumor removal; delayed primary re-excision and second-look surgery after initial chemotherapy are gaining more acceptance. Because of highmetastatic risk, systemic chemotherapy is also necessary. Novel agents are emerging which may alter the disease course in high-risk disease where the cure rate is still low. Radiation therapy is an important tool in the management of RMS and has gone through significant evolution during past four decades. This review will outline treatment strategies adopted in children RMS. The primary focus will be the North American approach with attention to advancements in radiation therapy, surgical techniques, and systemic therapies.
\end{abstract}

Keywords Rhabdomyosarcoma · Childhood tumors · Radiation therapy

\section{Introduction}

Rhabdomyosarcoma (RMS) is the most common soft tissue sarcoma in children. The treatment regimens have evolved

N. Esiashvili $(\bowtie) \cdot$ R. Prabhu $\cdot$ S. Kahn

Department of Radiation Oncology,

Winship Cancer Institute of Emory University, Atlanta, GA, USA

e-mail: nesiash@emory.edu

A. C. Paulino

Department of Radiation Oncology, The Methodist Hospital and

Texas Children's Hospital, Houston, TX, USA resulting in improved prognosis as a result of well-designed large cooperative group studies spanning over the course of four decades [1]. This prospective research has resulted in identification of different RMS risk groups and a risk-based multimodality approach leading to improved tumor control and survival [2]. The disease classification system is complex and based on both pretreatment clinical and radiographic data, as well as surgical and pathological findings. Balancing tumor local control with long-term functional and cosmetic morbidity remains as a major obstacle, especially in young children. Both surgery and radiotherapy (RT) play an essential role in local control. The goal of surgery is gross tumor removal without major functional or cosmetic compromise. Radiotherapy doses and targeted regions are based on areas at highest risk. The use of systemic therapy has made it possible to reduce radiation doses and volumes which benefits children at risk of long-term morbidity. The purpose of this review paper is to summarize current treatment strategies in Northern America in children diagnosed with RMS.

\section{Clinical presentation and biologic factors}

RMS accounts for $40 \%$ of all soft tissue sarcomas and $7 \%$ of all childhood malignancies diagnosed each year in the US [3]. This disease presents in a variety of anatomical sites with head and neck and genitourinary sites being the most predominant (Fig. 1a and b). Based on their histological make-up, tumors are divided into embryonal RMS (ERMS) $(57 \%)$, with botryoid and spindle cell variants, and alveolar RMS (ARMS) (23\%) [3]. Biological data is emerging on distinct genetic features of ERMS and ARMS which is proving to drive their clinical behavior. ARMS has a more aggressive clinical behavior with the highest risk for local and distant progression [4]. Two consistent chromosomal translocations are found in tumor tissue from the ARMS [5]. The more frequent translocation involves chromosome 13 and 

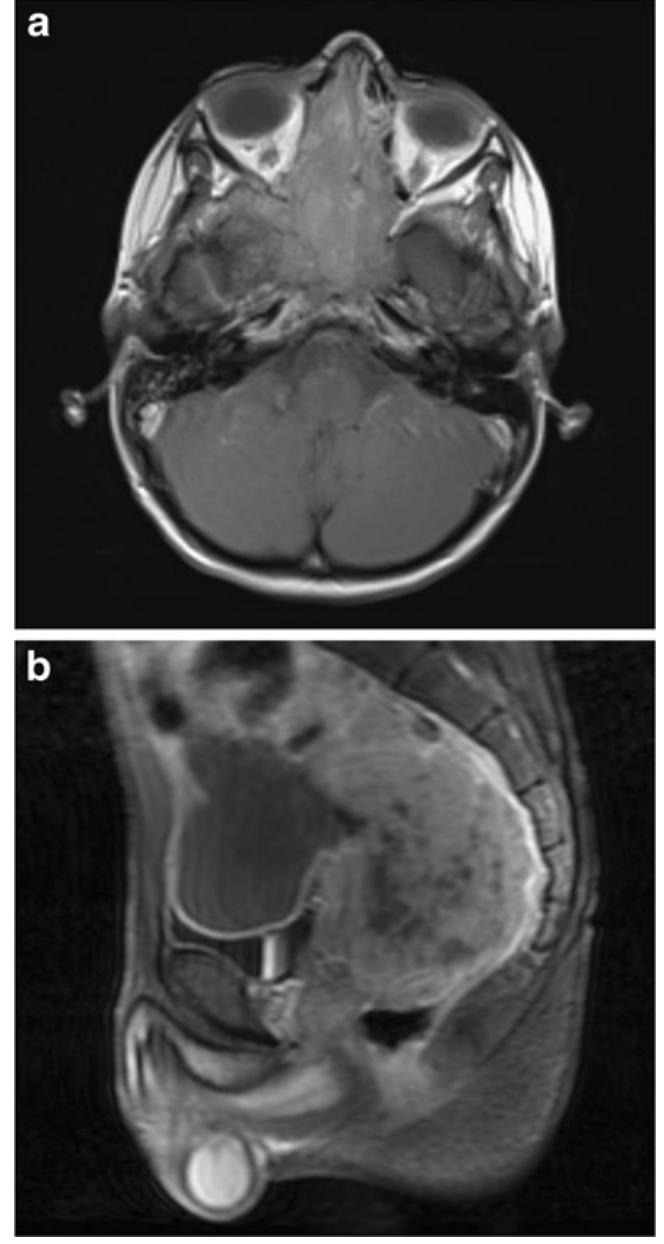

Fig. 1 a Heterogeneously enhancing mass centered within the nasal cavity with extension into sphenopalatine fossa, ethmoid sinuses and medial orbital walls. b Large heterogeneous mass in the pelvis arising posterior to the bladder and anterior to the rectum

$2[\mathrm{t}(2 ; 13)(\mathrm{q} 35 ; \mathrm{q} 14)]$, leading to a fusion product of the FKHR and PAX3 genes. An additional translocation involves chromosome 13 and $1[\mathrm{t}(1 ; 13)$ (p36; q14)], leading to a fusion product of the FKHR and the PAX7 genes. The presence of these translocations can serve as a useful diagnostic tool for predicting residual disease and outcome, with the t $(1 ; 13)$ having a better outlook compared to $t(2 ; 13)$.

\section{Prognostic factors, staging, and risk stratification}

As in most tumors, stage is one of the main prognostic factors. The International Rhabdomyosarcoma Study Group (IRSG) developed a surgical-pathologic grouping system based on extent of tumor removal from initial surgery (Table 1). The International Rhabdomyosarcoma Studies helped to define RMS risk groups, gain knowledge of disease biology and develop risk-adapted therapy. The International Rhabdomyosarcoma Study (IRS) protocols IRS-I, -II, and -III
Table 1 Intergroup rhabdomyosarcoma study group surgical-pathologic grouping system

\begin{tabular}{ll}
\hline Group & Definition \\
\hline I & $\begin{array}{c}\text { Localized tumor, completely removed with pathologically } \\
\text { clear margins and no regional lymph node involvement }\end{array}$ \\
II & $\begin{array}{c}\text { Localized tumor, grossly removed with (a) microscopically } \\
\text { involved margins, (b) involved, grossly resected regional } \\
\text { lymph nodes, or (c) both } \\
\text { III } \quad \begin{array}{c}\text { Localized tumor, with gross residual disease after grossly } \\
\text { incomplete removal, or biopsy only }\end{array} \\
\text { IV } \quad \text { Distant metastases present at diagnosis }\end{array}$ \\
\hline
\end{tabular}

prescribed treatment plans based on the surgical-pathologic grouping system which turned out to be highly predictive for disease outcome [6, 7]. The IRSG then incorporated a TNM pretreatment staging system based on primary site, tumor size, presence of absence of invasion into surrounding tissues, regional lymph node status and the distant metastasis status (Table 2) [8]. Currently, the Soft Tissue Sarcoma Committee of Children Oncology group (COG-STS) is incorporating both surgical-pathologic as well as the TNM staging system. Finally, tumor biology plays a significant impact on disease outcome with ARMS having a worse outcome compared to ERMS $[9,10]$. The RMS histological types are incorporated into a final COG-STS risk definition scheme (Table 3). It should be noted that undifferentiated sarcomas (UDS) are now treated on non-RMS COG-STS protocols.

There are additional factors that may influence outcome of RMS but currently are not incorporated into riskassignment. Age is a prognostic factor in patients with metastatic disease, particularly when combined with histology and biology $[5,11]$. Another group where age affects the disease outcome is in boys with paratesticular ERMS where older age ( $>10$ year of age) at diagnoses affects the rate of retroperitoneal lymph node involvement [12]. It is still controversial if extent of resection affects RMS outcome, but second-look surgery is gaining more role as it allows for reduction of RT dose [13-16]. Another

Table 2 Intergroup rhabdomyosarcoma study group presurgical staging system

\begin{tabular}{lllll}
\hline Stage & $\begin{array}{l}\text { Sites of } \\
\text { primary } \\
\text { tumor }\end{array}$ & $\begin{array}{l}\text { Tumor } \\
\text { size }(\mathrm{cm})\end{array}$ & $\begin{array}{l}\text { Regional } \\
\text { lymph } \\
\text { nodes }\end{array}$ & $\begin{array}{l}\text { Distant } \\
\text { metastases }\end{array}$ \\
\hline 1 & $\begin{array}{l}\text { Orbit, non-PM head/ } \\
\text { neck; GU non- } \\
\text { bladder/prostate; } \\
\text { biliary tract }\end{array}$ & Any size & N0, N1 & M0 \\
& $\begin{array}{l}\text { All other sites } \\
\text { All other sites }\end{array}$ & $\leq 5$ & N0 & M0 \\
3 & Any Site & N1 & N0 or N1 & M0 \\
4 & & Any size & N0 or N1 & M1 \\
\hline
\end{tabular}


Table 3 Current Children's Oncology Group (COG) risk groups

\begin{tabular}{llll}
\hline Histology & Clinical group & Stage & Risk group \\
\hline ERMS & I, II, III & 1 & Low (Subset A) \\
ERMS & I, II & 2,3 & Low (Subset b) \\
ERMS & III & 2,3 & Intermediate \\
ARMS & I, II, III & $1,2,3$ & Intermediate \\
ARMS & IV & 4 & High \\
ERMS & IV & 4 & High \\
\hline
\end{tabular}

interesting finding is the significance of tumor volume and patient weight as predictors of outcome in children with intermediate-risk RMS [17]. Prognostic significance of tumor response has been evaluated in clinical Group III patients on IRS-IV and have shown that the 5-year failure-free survival was similar for participants achieving complete response $(80 \%)$ versus partial or no response $(78 \%)$; resection of residual mass did not affect the outcome [18].

\section{Evolution of current therapy for RMS}

Current knowledge of appropriate treatment for RMS has evolved based on four decades of experience gained from well-designed clinical studies. The IRSG was formed in 1972 on the basis of three cooperative pediatric cancer treatment groups with the goal to investigate biology and therapy of RMS and UDS in children [19]. There were five consecutive clinical studies (IRS-I-V) with over 4,000 patients enrolled. Eligible patients were less than 21 years of age with newly diagnosed, untreated RMS and UDS. All pathology materials were reviewed centrally along with review of operative procedures and staging and grouping information by IRSG committee members. Additionally, independent agency the Quality Assurance Review Center (QARC, Providence, RI, USA) and IRSG radiation oncologists reviewed radiation oncology records.

The IRS-I stratified patients by group based on the extent of surgical resection and the presence or absence of distant metastases [6]. Radiation doses given were based on age and were between 40 to 60 Gray (Gy). It was demonstrated that Group I favorable histology patients have an excellent 5year failure-free survival (FFS) of $80 \%$ without RT. The conclusion was that local RT was not necessary for these low-risk Group I favorable histology patients. Other findings included no benefit with the addition of cyclophosphamide to vincristine and dactinomycin (VA) for Group II patients, with 5 year FFS of approximately $70 \%$ in both arms. In addition, Group III and IV patients did not benefit from the addition of doxorubicin to VA and cyclophosphamide (VAC) chemotherapy. The IRS-II introduced the concept of risk-adaptive RT dosing based on group, clinical factors, and age [20]. RT was excluded for Group I patients (not including extremity alveolar histology). Risk-adaptive RT dosing was as follows: Group I, no RT; Group II, 40 to $45 \mathrm{~Gy}$; Group III and $<5 \mathrm{~cm}$ and $<6$ years old, 40 to $45 \mathrm{~Gy}$, Group III and $>5 \mathrm{~cm}$ or $>6$ years old, 45 to $50 \mathrm{~Gy}$; Group III $>6$ years old and $>5 \mathrm{~cm}, 50-55 \mathrm{~Gy}$. Locoregional failure rates varied by group and histology: Group II $10 \%$, Group III $20 \%$, Group IV $41 \%$, favorable histology $13 \%$, unfavorable histology $41 \%$ [21]. Unlike the other clinical groups, the predominant pattern of failure for Group III patients was locoregional, making up $53 \%$ of all failures. The IRS-III continued to refine the risk-adaptive RT dose regimen [19]. The RT doses in IRS-III were as follows: Group I favorable histology - no RT; Group I unfavorable and Group II, 41.4 Gy; Group III, 41.4 Gy if $<5 \mathrm{~cm}$ and $<6$ years old, 45 Gy if $>5 \mathrm{~cm}$ or $>6$ years old, 50.4 Gy if both $>5 \mathrm{~cm}$ and $>6$ years old. The significant benefit for local control with RT for Group I unfavorable histology patients was confirmed [22]. Five-year actuarial local control for Group III patients receiving 41.4, 45, and 50.4 Gy were 14,23 , and $16 \%$, respectively. Though still unacceptably high, 50.4 Gy resulted in the lowest rate of local failure taking into account age and tumor size, and thus became the standard dose for Group III patients in upcoming trials. The IRS-IV posed the first randomized radiation therapy question since IRS-I [23]. In an attempt to increase local control for patients with Group III disease, patients were randomized to conventional 50.4 Gy (C-RT) versus a hyperfractionated regimen to 59.4 Gy given at 1.1 Gy BID (HF-RT) [24]. The RT for other clinical stages/groups represented further refinement of risk-adaptive dosing. Stage 1 or 2, Group I patients did not receive RT while Stage III, Group I and all Group II patients received 41.4 Gy. There were no differences between C-RT and HF-RT for Group III patients with 3-year actuarial failure-free survival of 73 and $83 \%$, and overall survival of 72 and $81 \%$, respectively. The 5year actuarial local and regional relapse for randomized patients was 13 and $3 \%$, respectively [23]. The IRS-V introduced risk categories based on stage, group, and histology in order to stratify patients as low, intermediate, or high risk based on outcomes analysis of patients on IRS-III and -IV [2]. The COG D9602 (IRS-V) prospectively investigated the ability to reduce RT dose for favorable histology, low-risk patients. RT dose was as follows: Group I no RT, Group IIA (positive microscopic margins) 36 Gy, Group IIB (resected involved lymph nodes) $41.4 \mathrm{~Gy}$, orbital location Group III 45 Gy, non-orbital location Group III 50.4 Gy. Second-look operation (SLO) at week 12 was allowed for certain non-orbital Group III tumors as an attempt to reduce the required dose of RT. RT doses after SLO: R0/R1 after SLO 36 Gy, R0/R1 resected involved lymph nodes 41.4 Gy, R2 resection or no SLO 50.4 Gy. Five-year local recurrence rates were $15 \%$ for favorable site Group IIA, $0 \%$ for 
unfavorable site Group IIA, and $14 \%$ for orbital location Group III [14]. Favorable site Group IIA and orbital Group III patients received VA chemotherapy while unfavorable site Group IIA patients received VAC chemotherapy. The conclusion from this study is that local control with reduced dose RT for low-risk patients is not compromised as long as cyclophosphamide is included in the chemotherapy regimen. The current low-risk study protocol ARST0331 continues these reduced doses, with all patients receiving intermediate doses of cyclophosphamide.

Intermediate-risk patients were treated on $\mathrm{COG}$ protocol D9803 (IRS-V). RT as local control was administered at week 12. Unfavorable histology Group I and Group IIA received 36 Gy. Group IIB and Group III patients received 41.4 Gy and 50.4 Gy, respectively. In a combined analysis with IRS-IV, it was found that $2.2 \%$ of patients experienced early disease progression prior to RT and did not receive planned protocol RT [24]. Nearly all early progression events $(95 \%)$ included progression at the local tumor site. This is the basis for RT at an earlier time point (week 4) on the current intermediate-risk ARSR0531 protocol. Current COG low-risk protocol (ARST0331) put cyclophosphamide back in initial 4 cycles of chemotherapy and kelp radiation dose to the orbit for Group III tumors at $45 \mathrm{~Gy}$, hoping to maintain high local control rate. The efficacy of this dose reduction will continue to be studied in the current COG low-risk RMS study, ARST0331.

\section{Local control considerations based on risk groups}

\section{Low-risk RMS}

Low-risk RMS group include patients with non-metastatic ERMS presenting in favorable sites (orbit, non-parameningeal head and neck, non-bladder/prostate genitourinary and biliary tract) and children with non-metastatic ERMS in unfavorable sites (bladder, prostate, extremity, parameningeal, etc.) who, at time of diagnosis, had undergone a gross total resection (Group I and II). Patient with ARMS, UDS, and ectomesenchymomas are excluded from this group.

The current challenge is maintaining or improving this outcome with less toxicity from multimodality treatments. Patients with ERMS in favorable sites (Stage 1) with Group I and II, as well as orbital RMS with Group III disease comprise a favorable subgroup of low-risk group and are found to have the best outcome with standard therapy. Additionally, current COG protocols have assigned Stage 2 Groups I and II ERMS to this category since they too were found to have a favorable outcome. It is expected that this subset of patients will have a 5-year FFS above $90 \%$. The second subset of low-risk patients consists of Stage 3, Groups I and II, and Stage 1 Group III non-orbit ERMS with less favorable disease and usually would receive VAC instead of VA chemotherapy for achieving a better outcome [25].

Surgical staging is part of the initial diagnostic process and is performed in all cases to obtain an adequate histologic specimen with the possibility for a delayed gross tumor resection. Clinical staging of regional lymph nodes, including radiography, is accepted for majority of primary sites, but regional lymph node sampling is required for all patients with the tumor primary site in the extremity. Children 10 years old or older with paratesticular primary tumors and negativestaging computed tomography images of the abdomen and pelvis are required to have a staging ipsilateral retroperitoneal lymph node dissection before entry into the study. A more aggressive surgical procedure is indicated if there is a chance of achieving a gross total resection upfront without compromising function or cosmesis. Patients with non-orbital Group III tumors can be evaluated for SLO at week 12 if gross total resection is possible without significant functional or cosmetic deficit. RT can be delivered after adequate healing from surgery.

Patient with ERMS Stage 1 and Group I have excellent tumor control rates after initial surgery and chemotherapy and do not require any RT. Wolden et al. analyzed the effect of RT on outcome according to tumor histology for patients treated on IRS-I, II, and III based on whether they actually did or did not receive RT [22]. For patients with ERMS, there was a trend toward improved FFS for the 27 patients who received RT compared with the 246 who did not receive RT, but the difference did not reach statistical significance $(P=0.10)$. Overall survival for the two groups was identical (approximately $95 \%$ at 10 years, $P=0.83$ ).

Postoperative RT is indicated only for postoperative residual gross or microscopic tumor. Group II patients and Group III patients with orbital tumors usually begin RT at week 3. Ninety percent of Group II received radiation therapy on Intergroup Rhabdomyosarcoma Study (IRS)-I to IRS-IV (1972 to 1997) [26]. Five-year FFS differed significantly by subgroup (IIa, $75 \%$; IIb, $74 \%$; IIc, $58 \%$; $P=$ 0.0037 ) and treatment (IRS-I, $68 \%$; IRS-II, $67 \%$; IRS-III, $75 \%$; IRS-IV, $87 \%$; $P<0.001$ ). Multivariate analysis revealed positive associations between primary site (favorable), histology (embryonal), subgroup IIa or IIb, treatment (IRS-III/IV), and better FFS rates. Local treatment failure was similar for patients with subgroup IIa (8\%), IIb (9\%), and IIc (6\%) and did not differ by histologic subtype. A total of 37 patients did not receive RT, although they were entered onto regimens that specified RT. Twenty of the 37 patients did not receive RT because of concerns for late effects in young children. Of these 20 patients, the 5-year FFS rate was $69 \%$ and the 5 -year OS rate was $85 \%$.

Children with Group IIA disease (microscopic residual viable tumor at the surgical margins), receive reduced RT 
dose to 36 Gy in 20 fractions. For Groups IIB or IIC patients (involved lymph nodes without [B] or with [C] microscopic positive surgical margins), $41.4 \mathrm{~Gy}$ in 23 fractions is indicated. Children with Group III orbital tumors are currently treated with 45 Gy in 25 fractions. This dose reduction from standard Group III dose of 50.4 Gy is feasible if adequate chemotherapy (cyclophosphamide containing) is given. Children with non-orbital Group III primary tumors who did not undergo SLO should receive 50.4 Gy in 28 fractions. Children who undergo SLO receive RT according to the extent of the resection: with negative lymph nodes who had a gross total resection with or without microscopically negative margins receive 36 Gy in 20 fractions; patients with involved lymph nodes but no gross tumor receive 41.4 Gy in 23 fractions, and patient with gross residual tumor receive 50.4 Gy in 28 fractions [14, 25].

The local control approach for girls with non-resected vaginal RMS enrolled onto IRSG/COG studies has differed from that used at other primary sites by delaying or eliminating RT based on response achieved with chemotherapy and delayed primary resection. Based on analysis of IRS-V (D9602) and ARST0331 revealed that these girls had high rates of local recurrence (LR) (5-year LR $26 \%$ on D9602 and 2-year LR $43 \%$ on ARST0331) which appear to correlate with decrease in cumulative doses of cyclophosphamide in chemotherapy regimen and lack of RT use; current recommendation is to apply RT indications similar to other sites [27].

\section{Intermediate-risk RMS}

Patients at intermediate risk are those with localized ARMS, stages 1 through 3, or ERMS, stages 2 and 3 with gross residual disease, or ERMS, Stage 4 younger than 10 years of age. IRS studies have failed to demonstrate improved outcome of these challenging patients despite of incorporation of various chemotherapy agents [28, 29]. Early results have shown that the best response rates have incorporated vincristine and irinotecan (VI); in the current COG study (ARST0531) for intermediate-risk RMS, patients are randomized to VAC versus VAC alternating with VI for 43 weeks [30].

Local therapy plays an important role in this group of RMS patients. Upfront surgical resection is attempted, when feasible and non-mutilating. Second-look operation is also encouraged for initially unresected tumors for selected anatomic sites, including extremity, dome of bladder, and trunk which should help reduce RT dose. RT plays a particularly important role in unfavorable histology RMS. On IRS-I and -II, patients with ARMS or UDS who received RT compared with those who did not receive RT had greater 10-year FFS rates $(73 \%$ versus $44 \% ; P=0.03)$ and overall survival rates (82\% versus $52 \% ; P=0.02$ ) [22]. Similar findings were observed on combined results from IRS-III and -IV [31]. This prompted moving early stage ARMS (Stage 1 and Group I) from the low-risk protocol (D9602) to intermediate-risk study (D9803) in IRS-V. Additionally, an analysis from IRSIV revealed a subgroup of patients with Stage 4, Group IV ERMS ( $<10$ years old) which had a more favorable prognosis and treated as intermediate-risk patients in IRS-V (D9803) [10]. However, this group was moved back to high-risk category on the current COG protocol.

Almost half of patients with RMS would present with Group III tumors, notably in parameningeal sites where surgical resection is not a viable option. Definitive RT plays a critical role and can achieve a good local control rate. Dose escalation with hyperfractionation was tested in IRS-IV study and did not improve tumor control [23]. As mentioned above, tumor response at the end of therapy in clinical Group III patients was found to have no adverse effect on local control or survival and aggressive salvage treatment options, like resection of residual mass or alternative chemotherapy regimens should be avoided [18].

Local therapy is targeted around week 12. Currently RT doses for ARMS are as follows: 36 Gy for Group I; 36 Gy for Group II node negative; 41.4 Gy for Group II node positive; and 50.4 Gy for Group III. Patients with ERMS Group III (excluding orbital treated on low-risk protocols), are treated with 50.4 Gy dose.

\section{High-risk RMS}

Patients with Stage 4 and Group IV metastatic RMS tumors account for only $16 \%$ of all RMS cases. Historically, this group of patients had done poorly without much progress over the past three decades despite the use of more intensive therapies [32]. Again, IRS-IV data confirmed that Stage 4 patients with ERMS who are $>10$ years of age and those with ARMS have very poor outcome and should be treated as high risk [10].

Novel chemotherapy and targeted drugs are currently being investigated in high-risk patients. Two consecutive COG Phase II tested upfront window of irinotecan alone or in combination with vincristine. The irinotecan trial was closed early because of a high progression rate (32\%), however, the VI arm had an $8 \%$ progression rate with an overall $70 \%$ response rate [30]. The recently completed COG study ARST0431 tested the tolerability and early outcome data from an intensive combination of VI, intervalcompressed vincristine/doxorubicin/cyclophosphamide alternating with ifosfamide/etoposide in addition to VAC chemotherapy in high-risk RMS. Results have not been reported at this time. The successor COG study for high-risk RMS, ARST08P1, uses the same backbone with the addition of the novel therapeutic agent cetuximab and/or temozolomide in a series of three sequential pilot studies. 
Local control in the setting of metastatic disease is delayed to allow delivery of more systemic therapy. Patients, with exception of emergent treatments, usually will initiate RT at week 20 of chemotherapy. Both primary tumors and metastatic sites are targeted; however, if multiple sites prohibit simultaneous treatment, second course of RT can be delivered in later part of therapy, usually around week 47 of chemotherapy. Aggressive resection is not commonly pursued because of widespread disease and radiotherapy is viewed as the mainstay local therapy. The RT dose to primary site is selected according to the clinical/pathological grouping and metastatic sites are treated to a definitive dose as well. A cone-down boost may be feasible after 36 Gy if a substantial decrease in tumor size is seen in response to chemotherapy.

\section{Radiotherapy targets and techniques}

Historically, RT for RMS applied generous target volumes and doses; however, data gained from early IRS experience helped to refine RT techniques. Modern megavoltage radiation and image-based methods allow delivery of higher quality radiotherapy. RT quality and compliance does play an essential role in disease outcome as it was shown based on recent analysis of IRS and COG protocols: $55 \%$ of patients with operative bed recurrence did not receive the intended RT; the most frequent deviation being RT omission (41\%), followed by dose (38\%) and/or volume (20\%) deviation [33].

The RT target volumes are usually defined in accordance with the International Commission on Radiation Units and Measurements 50 guidelines [34]. The gross tumor volume (GTV) is defined as the preoperative and pre-chemotherapy extent of tumor based on diagnostic imaging and clinical examination. GTV can be modified if a tumor volume returned to normal anatomic position retracted from adjacent anatomical structures, like bowel or lung which it had originally displaced but not infiltrated. This usually happens as a result of surgery and/or chemotherapy. On the other hand, structures like pleura, peritoneum, and mesentery should be included in GTV in case they were initially involved infiltrated with tumor. The clinical target volume (CTV) includes the GTV and sites with potential occult tumor involvement including lymph nodes adjacent to the GTV that may be clinically involved and is usually created with expansion of the GTV by 1 to $1.5 \mathrm{~cm}$. If regional lymph nodes were involved, the CTV should include the entire lymph node chain. The CTV is anatomically modified to avoid extension outside anatomically confined structures and outside the body if tumor was not infiltrating into these areas at time of diagnosis. Planning target volume is usually defined as CTV plus an institutional-specified margin to account for day-to-day setup variation related to the ability to immobilize the patient and physiologic motion of the CTV, usually a minimum $0.5 \mathrm{~cm}$ margin. Patients receiving 36 or 41.4 Gy total dose should not have original CTV adjusted, however, for patients who demonstrate rapid reduction or Group III tumor who did not undergo SLO and had more pushing rather than infiltrating tumor margins, a cone-down boost can be considered after 36-41.4 Gy and is not associated with inferior disease local control $[35,36]$. Intensity modulated radiation therapy (IMRT), volumetric modulated arc therapy (VMAT), tomotherapy, and proton beam therapy are gaining popularity with the goal of sparing normal structures [37-42].

RMS in parameningeal sites presents a unique anatomical challenge because of their proximity to multiple organs at risk (optic apparatus, hypothalamic-pituitary axis, brain, musculoskeletal structures, lacrimal and salivary glands, etc.). Novel conformal RT techniques like IMRT, VMAT, tomotherapy, and proton beams are especially important for this challenging site and should allow reducing doses to the majority of these structures [36, 39-41, 43] (Fig. 2). Another strategy for a decline in treatment toxicity is reduction of irradiated tissues based on treatment response [36]. Particular attention must be paid to careful evaluation of extent of cranial invasion. Patients with frank intracranial invasion may benefit from early initiation of RT (within 0-2 weeks) as suggested from initial IRS data [44], however, others have presented conflicting data [45].

Patients presenting with wide tumor dissemination present unique challenges. In patients with pulmonary metastasis, regardless of initial tumor resection and/or response to chemotherapy, whole lung irradiation is indicated to $15 \mathrm{~Gy}$ at 1.5 Gy per fraction [46]. In patients with metastatic

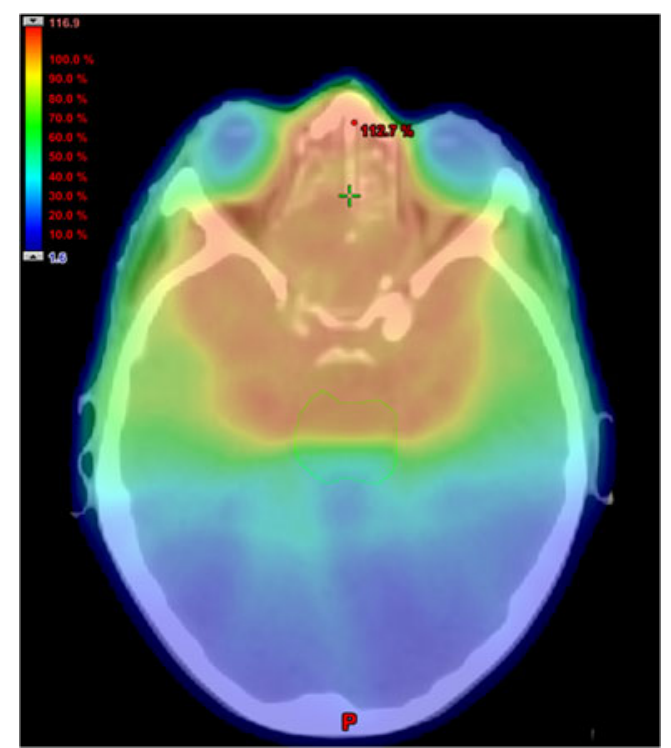

Fig. 2 VMAT plan for parameningeal RMS: axial computer tomography of involved site demonstrating dose color-wash 
disease to the abdominal cavities, i.e., diffuse peritoneal implants or malignant ascites, whole abdomen RT is recommended with doses around 24 Gy at 1.5 Gy with utilization of appropriate kidney and liver shielding techniques. Sequential boost to residual gross tumor volume to total 50.4 Gy can be planned, when feasible.

In general, lymph node spread is uncommon in RMS, but can be seen more often in extremity and paratesticular tumors, particularly in older boys [47-49]. Sentinel lymph node sampling is recommended for RMS in extremities, especially with alveolar histology. In paratesticular RMS, lymph node dissection is important for boys $>10$ years of age regardless of radiographic findings and in $<10$ year olds if enlarged lymph nodes are found on CT. For head and neck tumors, careful attention must be paid to neck imaging and there is emerging data on utility of PET in detecting subclinical disease. Only in the case of proven lymph node involvement should the regional nodes be included in clinical target volume. There is still lack of evidence on benefit of treating ipsilateral pelvic lymph nodes in patients with paratesticular primary tumor who will undergo para-aortic lymph node irradiation.

Brachytherapy is an attractive modality for sarcomas because of better preservation of the adjacent uninvolved tissues; however, data remains limited. Historically, low dose-rate brachytherapy was more commonly used for intracavitary and interstitial applications [50,51], however, highdose and pulse rate methods are gaining more acceptance due to less need for immobilization and isolation [52-55] (Fig. 3).

Choosing a proper local control modality is very challenging in infants, particularly in patients younger than 2-years of age, because they are at increased risk for long-term morbidity

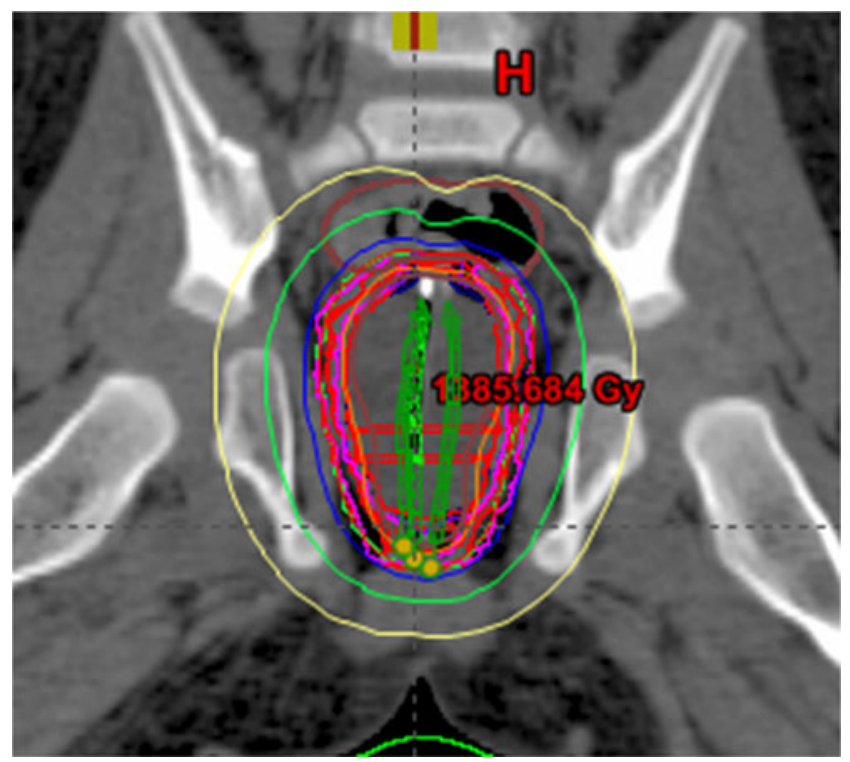

Fig. 3 Endocavitary high dose-rate brachytherapy for vaginal RMS of RT or aggressive surgery. Many clinicians are uncomfortable treating very young children with standardized local control guidelines and commonly decrease radiotherapy dose. This unfortunately does result in inferior tumor local control and ultimately poor survival [56]. There is limited data on RT outcomes in infants. Normal-tissue-sparing techniques such as IMRT, proton beams and intraoperative radiation therapy or brachytherapy are encouraged $[51,56,57]$.

\section{Toxicity}

Radiotherapy toxicities are site specific. The most common acute toxicities in RMS patients receiving concurrent RT and chemotherapy are radiation dermatitis, mucositis, and myelosupression. Usually, side effects are cumulative over the course of 4 to 6 weeks of irradiation are exacerbated by concurrent chemotherapy [58]. Because of concerns for radiation sensitization, dactinomycin usually is given before beginning of RT and is withheld during RT.

Long-term treatment complications can occur in up to a third of patients [59]. Musculoskeletal hypoplasia is expected in younger patients and should correlate closely with the bone and muscle volume treated as well as patient's age. Craniofacial hypoplasia is expected to be more pronounced in patients treated to head and neck sites, particularly in infants $[59,60]$. Limb-length discrepancy and decreased circumference is expected in young children receiving RT for extremity RMS. Soft tissue fibrosis and lymphedema is also a major concern, particularly in patients receiving surgical intervention in conjunction with RT.

Neuroendocrine issues are common in children treated for parameningeal RMS and unfortunately are often overlooked as many children do not go through routine screening [61]. Growth hormone is the most susceptible to radiation effects at relatively low dose levels. Other hormones, like thyroid stimulating hormone, gonadotropin, and corticotrophin hormones can be reduced as well and need close monitoring with possible replacement therapy. Neuroendocrine complications can be reduced with modern techniques such as IMRT or proton beam [39, 40, 43]. Ocular complications are avoidable in most cases given the maximum dose level prescribed for orbital and other head and neck primary tumors. While cataract formation, keratopathy, retinopathy, and dry eye can occur at doses up to $50.4 \mathrm{~Gy}$, loss of useful vision is very rare $[62,63]$. Tumors in proximity to cochlea often pose a challenge for hearing preservation.

Tumors in the thoracic region may present as a challenge for achieving dose constraints for the lungs and heart, while for abdominal tumors, the liver, kidneys, and bowel are the organs at risk. Modern conformal methods like IMRT, VMAT or proton beams should allow better sparing of these tissues. Pelvic tumors are usually in proximity with bladder, 
rectum and reproductive organs and make it very difficult to avoid significant risks for late organ dysfunction [64-68].

The incidence of second malignant neoplasm (SMN) in children treated for RMS has been studied in patients enrolled onto IRS-I and -II [69]. In 1,770 patients with primary RMS treated between 1972 and 1984, 22 developed a SMN. The most common tumor type was a bone sarcoma followed by acute nonlymphoblastic leukemia. The median time to the development of an SMN was 7 years (range, 1 to 16 years). The 10-year cumulative incidence rate was $1.7 \%$ for both studies. The cumulative incidence estimates were highest for patients who received both an alkylating agent and radiotherapy. The majority of patients for whom family histories were available had either neurofibromatosis or a family history that suggested the Li-Fraumeni syndrome. The results of this study suggest that genetic abnormalities play a prominent role in the development of an SMN after therapy for a primary RMS.

\section{Conclusions}

RMS is the most common soft tissue sarcoma affecting patients in first two decades of life. This disease poses unique anatomical and functional challenges for local control especially in very young children. Systemic chemotherapy plays an essential role in RMS treatment because of its high metastatic potential. While there have been modest improvements in patients' survival over the course of the last four decades, intermediate and high-risk RMS still face major risk of disease progression and death. Despite major improvements in both surgical and radiotherapy techniques, patients continue to face significant treatment toxicities. Novel therapeutic options are currently being explored to improve survival and overall functional outcome of patients affected by RMS.

Conflict of interests Authors have no conflict of interests to disclose.

\section{References}

1. Malempati S, Hawkins DS (2012) Rhabdomyosarcoma: review of the Children's Oncology Group (COG) Soft-Tissue Sarcoma Committee experience and rationale for current COG studies. Pediatr Blood Cancer 59(1):5-10

2. Meza JL et al (2006) Analysis of prognostic factors in patients with nonmetastatic rhabdomyosarcoma treated on intergroup rhabdomyosarcoma studies III and IV: the children's oncology group. J Clin Oncol 24(24):3844-3851

3. Ognjanovic $\mathrm{S}$ et al (2009) Trends in childhood rhabdomyosarcoma incidence and survival in the United States, 1975-2005. Cancer 115(18):4218-4226
4. Newton WA Jr et al (1988) Histopathology of childhood sarcomas, Intergroup Rhabdomyosarcoma Studies I and II: clinicopathologic correlation. J Clin Oncol 6(1):67-75

5. Kelly KM et al (1997) Common and variant gene fusions predict distinct clinical phenotypes in rhabdomyosarcoma. J Clin Oncol 15 (5):1831-1836

6. Maurer HM et al (1988) The intergroup rhabdomyosarcoma studyI. A final report Cancer 61(2):209-220

7. Crist WM et al (1990) Prognosis in children with rhabdomyosarcoma: a report of the intergroup rhabdomyosarcoma studies I and II intergroup rhabdomyosarcoma committee. J Clin Oncol 8 (3):443-452

8. Lawrence W Jr et al (1997) Pretreatment TNM staging of childhood rhabdomyosarcoma: a report of the intergroup rhabdomyosarcoma study group. Children's cancer study group. Pediatric Oncology Group Cancer 80(6):1165-1170

9. Rodeberg DA et al (2011) Prognostic significance and tumor biology of regional lymph node disease in patients with rhabdomyosarcoma: a report from the children's oncology group. J Clin Oncol 29(10):1304-1311

10. Raney RB et al (2001) Rhabdomyosarcoma and undifferentiated sarcoma in the first two decades of life: a selective review of intergroup rhabdomyosarcoma study group experience and rationale for Intergroup Rhabdomyosarcoma Study V. J Pediatr Hematol Oncol 23(4):215-220

11. Breneman JC et al (2003) Prognostic factors and clinical outcomes in children and adolescents with metastatic rhabdomyosarcoma-a report from the Intergroup Rhabdomyosarcoma Study IV. J Clin Oncol 21(1):78-84

12. Wiener ES et al (2001) Controversies in the management of paratesticular rhabdomyosarcoma: is staging retroperitoneal lymph node dissection necessary for adolescents with resected paratesticular rhabdomyosarcoma? Semin Pediatr Surg 10(3):146-152

13. Hayes-Jordan A et al (2008) The impact of surgical excision in chest wall rhabdomyosarcoma: a report from the children's oncology group. J Pediatr Surg 43(5):831-836

14. Breneman J et al (2012) Local control with reduced-dose radiotherapy for low-risk rhabdomyosarcoma: a report from the Children's Oncology Group D9602 study. Int J Radiat Oncol Biol Phys 83(2):720-726

15. Raney B et al (2010) Impact of tumor viability at second-look procedures performed before completing treatment on the Intergroup Rhabdomyosarcoma Study Group protocol IRS-IV, 19911997: a report from the children's oncology group. J Pediatr Surg 45 (11):2160-2168

16. Blakely ML (1999) Does debulking improve survival rate in advanced-stage retroperitoneal embryonal rhabdomyosarcoma. J Pediatr Surg 34(5):736-741, discussion 741-2

17. Rodeberg D A, et al (2010) Tumor volume and patient weight as predictors of outcome in children with intermediate risk rhabdomyosarcoma: a report from the children's oncology group. Cancer

18. Rodeberg DA et al (2009) Prognostic significance of tumor response at the end of therapy in group III rhabdomyosarcoma: a report from the children's oncology group. J Clin Oncol 27 (22):3705-3711

19. Raney RB et al (2001) The intergroup rhabdomyosarcoma study group (IRSG): major lessons from the IRS-I through IRS-IV studies as background for the current IRS-V treatment protocols. Sarcoma 5(1):9-15

20. Maurer HM et al (1993) The intergroup rhabdomyosarcoma studyII. Cancer 71(5):1904-1922

21. Wharam MD et al (1997) Radiation therapy for rhabdomyosarcoma: local failure risk for clinical group III patients on intergroup rhabdomyosarcoma study II. Int J Radiat Oncol Biol Phys 38 (4):797-804 
22. Wolden SL et al (1999) Indications for radiotherapy and chemotherapy after complete resection in rhabdomyosarcoma: a report from the Intergroup Rhabdomyosarcoma Studies I to III. J Clin Oncol 17(11):3468-3475

23. Donaldson SS et al (2001) Results from the IRS-IV randomized trial of hyperfractionated radiotherapy in children with rhabdomyosarcoma-a report from the IRSG. Int J Radiat Oncol Biol Phys 51(3):718-728

24. Minn AY et al (2010) Early treatment failure in intermediate-risk rhabdomyosarcoma: results from IRS-IV and D9803-a report from the Children's Oncology Group. J Clin Oncol 28(27):42284232

25. Raney RB et al (2011) Results of the Intergroup Rhabdomyosarcoma Study Group D9602 protocol, using vincristine and dactinomycin with or without cyclophosphamide and radiation therapy, for newly diagnosed patients with low-risk embryonal rhabdomyosarcoma: a report from the Soft Tissue Sarcoma Committee of the Children's Oncology Group. J Clin Oncol 29(10):13121318

26. Smith LM et al (2001) Which patients with microscopic disease and rhabdomyosarcoma experience relapse after therapy? A report from the soft tissue sarcoma committee of the children's oncology group. J Clin Oncol 19(20):4058-4064

27. Walterhouse DO et al (2011) Local control and outcome in children with localized vaginal rhabdomyosarcoma: a report from the Soft Tissue Sarcoma committee of the Children's Oncology Group. Pediatr Blood Cancer 57(1):76-83

28. Crist WM et al (2001) Intergroup rhabdomyosarcoma study_-IV: results for patients with nonmetastatic disease. J Clin Oncol 19 (12):3091-3102

29. Arndt CA et al (2009) Vincristine, actinomycin, and cyclophosphamide compared with vincristine, actinomycin, and cyclophosphamide alternating with vincristine, topotecan, and cyclophosphamide for intermediate-risk rhabdomyosarcoma: children's oncology group study D9803. J Clin Oncol 27(31):5182-5188

30. Pappo AS et al (2007) Two consecutive phase II window trials of irinotecan alone or in combination with vincristine for the treatment of metastatic rhabdomyosarcoma: the Children's Oncology Group. J Clin Oncol 25(4):362-369

31. Raney RB et al (2010) Treatment results for patients with localized, completely resected (Group I) alveolar rhabdomyosarcoma on Intergroup Rhabdomyosarcoma Study Group (IRSG) protocols III and IV, 1984-1997: a report from the Children's Oncology Group. Pediatr Blood Cancer 55(4):612-616

32. Crist $W$ et al (1995) The third intergroup rhabdomyosarcoma study. J Clin Oncol 13(3):610-630

33. Million $L$ et al (2011) Influence of noncompliance with radiation therapy protocol guidelines and operative bed recurrences for children with rhabdomyosarcoma and microscopic residual disease: a report from the Children's Oncology Group. Int J Radiat Oncol Biol Phys 80(2):333-338

34. Monti AF et al (1995) An ICRU 50 radiotherapy treatment chart. Radiother Oncol 35(2):145-150

35. Chen $\mathrm{C}$ et al (2003) Volumetric considerations in radiotherapy for pediatric parameningeal rhabdomyosarcomas. Int J Radiat Oncol Biol Phys 55(5):1294-1299

36. McDonald MW et al (2008) Intensity-modulated radiotherapy with use of cone-down boost for pediatric head-and-neck rhabdomyosarcoma. Int J Radiat Oncol Biol Phys 72(3):884-891

37. Combs SE et al (2007) Intensity modulated radiotherapy (IMRT) and fractionated stereotactic radiotherapy (FSRT) for children with head-and-neck-rhabdomyosarcoma. BMC Cancer 7:177

38. Lin C (2012) Effect of radiotherapy techniques (IMRT vs. 3DCRT) on outcome in patients with intermediate-risk rhabdomyosarcoma enrolled in COG D9803 - a report from the Children's Oncology Group. Int J Radiat Oncol Biol Phys 82(5):1764-1770
39. Wolden SL et al (2005) Intensity-modulated radiotherapy for headand-neck rhabdomyosarcoma. Int J Radiat Oncol Biol Phys 61 (5):1432-1438

40. Childs SK et al (2012) Proton radiotherapy for parameningeal rhabdomyosarcoma: clinical outcomes and late effects. Int J Radiat Oncol Biol Phys 82(2):635-642

41. Kozak KR et al (2009) A dosimetric comparison of proton and intensity-modulated photon radiotherapy for pediatric parameningeal rhabdomyosarcomas. Int J Radiat Oncol Biol Phys 74(1):179186

42. Cotter SE et al (2011) Proton radiotherapy for pediatric bladder/ prostate rhabdomyosarcoma: clinical outcomes and dosimetry compared to intensity-modulated radiation therapy. Int $\mathrm{J}$ Radiat Oncol Biol Phys 81(5):1367-1373

43. Yang JC et al (2012) Intensity modulated radiation therapy with dose painting to treat rhabdomyosarcoma. Int J Radiat Oncol Biol Phys 84(3):e371-e377

44. Michalski JM et al (2004) Influence of radiation therapy parameters on outcome in children treated with radiation therapy for localized parameningeal rhabdomyosarcoma in Intergroup Rhabdomyosarcoma Study Group trials II through IV. Int J Radiat Oncol Biol Phys 59(4):1027-1038

45. Douglas JG, Arndt CA, Hawkins DS (2007) Delayed radiotherapy following dose intensive chemotherapy for parameningeal rhabdomyosarcoma (PM-RMS) of childhood. Eur J Cancer 43(6):10451050

46. Dantonello TM et al (2011) Embryonal rhabdomyosarcoma with metastases confined to the lungs: report from the CWS Study Group. Pediatr Blood Cancer 56(5):725-732

47. La TH et al (2011) Regional nodal involvement and patterns of spread along in-transit pathways in children with rhabdomyosarcoma of the extremity: a report from the Children's Oncology Group. Int J Radiat Oncol Biol Phys 80(4):1151-1157

48. La TH et al (2009) Local therapy for rhabdomyosarcoma of the hands and feet: is there a role for amputation? A report from the children's oncology group. Int J Radiat Oncol Biol Phys 75(3): S40-S41

49. Paulino AC, Pappo A (2009) Alveolar rhabdomyosarcoma of the extremity and nodal metastasis: is the in-transit lymphatic system at risk. Pediatr Blood Cancer 53(7):1332-1333

50. Flamant $F$ et al (1990) Long-term sequelae of conservative treatment by surgery, brachytherapy, and chemotherapy for vulval and vaginal rhabdomyosarcoma in children. J Clin Oncol 8(11):18471853

51. Magne $\mathrm{N}$ et al (2008) Vulval and vaginal rhabdomyosarcoma in children: update and reappraisal of Institut Gustave Roussy brachytherapy experience. Int J Radiat Oncol Biol Phys 72(3):878-883

52. Nag S, Grecula J, Ruymann FB (1993) Aggressive chemotherapy, organ-preserving surgery, and high-dose-rate remote brachytherapy in the treatment of rhabdomyosarcoma in infants and young children. Cancer 72(9):2769-2776

53. Abramson DH et al (1997) Implant brachytherapy: a novel treatment for recurrent orbital rhabdomyosarcoma. J AAPOS 1(3):154-157

54. Blank LE et al (2009) The AMORE protocol for advanced-stage and recurrent nonorbital rhabdomyosarcoma in the head-and-neck region of children: a radiation oncology view. Int J Radiat Oncol Biol Phys 74(5):1555-1562

55. Blank LE et al (2010) Brachytherapy as part of the multidisciplinary treatment of childhood rhabdomyosarcomas of the orbit. Int $\mathrm{J}$ Radiat Oncol Biol Phys 77(5):1463-1469

56. Puri DR et al (2006) The challenging role of radiation therapy for very young children with rhabdomyosarcoma. Int J Radiat Oncol Biol Phys 65(4):1177-1184

57. Malempati $\mathrm{S}$ et al (2011) Rhabdomyosarcoma in infants younger than 1 year: a report from the Children's Oncology Group. Cancer 117(15):3493-3501 
58. Gupta AA et al (2012) Patterns of chemotherapy-induced toxicities in younger children and adolescents with rhabdomyosarcoma: a report from the children's oncology group soft tissue sarcoma committee. Cancer 118(4):1130-1137

59. Paulino AC et al (2000) Long-term effects in children treated with radiotherapy for head and neck rhabdomyosarcoma. Int J Radiat Oncol Biol Phys 48(5):1489-1495

60. Krasin MJ et al (2012) Jaw dysfunction related to pterygoid and masseter muscle dosimetry after radiation therapy in children and young adults with head-and-neck sarcomas. Int $\mathrm{J}$ Radiat Oncol Biol Phys 82(1):355-360

61. Snyers A et al (2009) Malignant tumors of the nasal cavity and paranasal sinuses: long-term outcome and morbidity with emphasis on hypothalamic-pituitary deficiency. Int J Radiat Oncol Biol Phys 73(5):1343-1351

62. Gandhi PD et al (2011) Ophthalmic complications following treatment of paranasal sinus rhabdomyosarcoma in comparison to orbital disease. Ophthal Plast Reconstr Surg 27(4):241-246

63. Raney RB et al (2000) Late effects of therapy in 94 patients with localized rhabdomyosarcoma of the orbit: report from the intergroup rhabdomyosarcoma study (IRS)-III, 1984-1991. Med Pediatr Oncol 34(6):413-420
64. Macedo A Jr et al (2010) Sexual function in teenagers after multimodal treatment of pelvic rhabdomyosarcoma: a preliminary report. J Pediatr Urol 6(6):605-608

65. Gruschow K et al (2007) Surgical repositioning of the contralateral testicle before irradiation of a paratesticular rhabdomyosarcoma for preservation of hormone production. Pediatr Hematol Oncol 24 (5):371-377

66. Arndt $\mathrm{C}$ et al (2004) Does bladder preservation (as a surgical principle) lead to retaining bladder function in bladder/prostate rhabdomyosarcoma? Results from intergroup rhabdomyosarcoma study IV. J Urol 171(6 Pt 1):2396-2403

67. Cowles RA, Gewanter RM, Kandel JJ (2007) Ovarian repositioning in pediatric cancer patients: flexible techniques accommodate pelvic radiation fields. Pediatr Blood Cancer 49(3):339341

68. Raney B Jr et al (1993) Sequelae of treatment in 109 patients followed for 5 to 15 years after diagnosis of sarcoma of the bladder and prostate. A report from the Intergroup Rhabdomyosarcoma Study Committee. Cancer 71(7):2387-2394

69. Heyn R et al (1993) Second malignant neoplasms in children treated for rhabdomyosarcoma. Intergroup Rhabdomyosarcoma Study Committee. J Clin Oncol 11(2):262-270 